7. Schneider, M. F. \& Chandler, W. K. Nature 242, 244247 (1973).

8. Shirokova, N. \& Rios, E. J. Physiol (Lond) 502, 3-11 (1997).

9. Gonzalez, A. et al. J. Gen. Physiol. 115, 139-158 (2000).
10. Felder, E. \& Franzini-Armstrong, C. Proc. Natl Acad. Sci. USA 99, 1695-1700 (2002).

11. Shirokova, N., Garcia, J. \& Rios, E. J. Physiol. (Lond) 512, 377-384 (1998).

12. Conklin, M. W. Barone, V., Sorrentino, V. \& Coronado, R. Biophys. J. 77, 1394-1403 (1999).
13. Wang, X. et al. Nature Cell Biol. 7, 525-530 (2005)

14. Allen, D. G. Clin. Exp. Pharmacol. Physiol. 31, 485493 (2004).

15. Menke, A. \& Jockusch, H. Nature 349, 69-71 (1991).

\section{IRF-7 triggers the interferon alarm}

The type-I interferon (IFN- $\alpha / \beta)$ response is the immune system's preferred weapon against viral infections. It can be triggered in many cell types by the cytosolic detection of viral infection, or in differentiated plasmacytoid dendritic cells (pDCs) through the activation of Tolllike receptor 9 (TLR-9). In two recent papers in Nature (doi:10.1038/ nature03464 and 10.1038/nature03547) Honda et al. report on how, while investigating the in vivo role of the transcription factor IRF-7, they discovered that IRF-7 is critical to the IFN- $\alpha / \beta$ response in both virally infected cells and TLR-activated pDCs. The authors also describe how they have unveiled a mechanism for robust IFN production that is unique to $\mathrm{pDCs}$.

Using embryonic fibroblasts that were isolated from $\operatorname{Irf7} 7^{-/-}$mice, the authors observed that IRF-7, but not the adaptor MyD88, was required for the induction of IFN- $\alpha / \beta$ in response to viral infection, and that $\operatorname{Irf7^{-1-}}$ mice are more vulnerable to viral infections compared with mice that lack $\operatorname{Irf3}$ or Myd88 genes. In contrast, robust interferon production in pDCs through TLR-9 subfamily activation - either by viral infection or by the CpG-A ligand - depends on both IRF-7 and MyD88, and is independent of the previously implicated transcription factors IRF-1, -3 and -5 , and Smad3. The authors also demonstrate an essential role for the MyD88-IRF-7 pathway in pDCs, in the TLR-9-mediated induction of the antigen-specific $\mathrm{CD}^{+} \mathrm{T}$-cell response, which is regulated by IFN production. This result suggests that IRF-7 governs the entire interferondependent defence mechanism.

The details of IRF-7 activation remain unclear. Honda et al. do, however, offer an interesting explanation for how pDCs, and not other cells, are able to activate the MyD88-dependent IFN induction pathway. Fluorescence microscopy studies revealed that in pDCs the TLR-9 ligand CpG-A localizes with IRF-7 to the endosomal compartment, whereas in conventional dendritic cells (cDCs) it localizes to lysosomes. Similarly, in macrophages - cells that are unable to produce IFN - CpG-A did not colocalize with the MyD88-IRF-7 complex in granule structures,

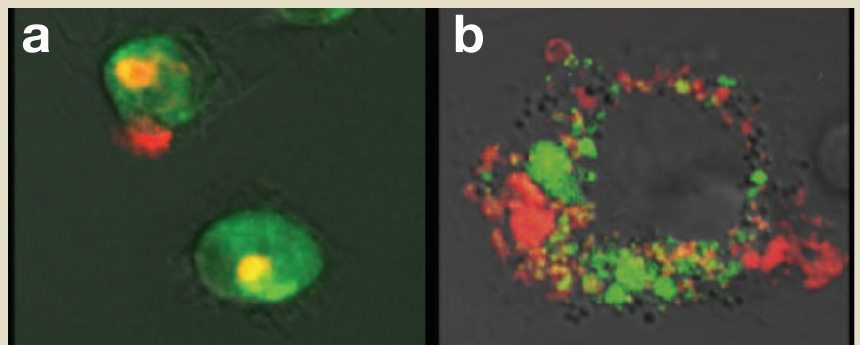

The TLR-9 ligand CpG-A (red) colocalizes with IRF-7 (green) in endosomal structures of pDCs (a), but fails to do so in CDCs (b). Figure reproduced with permission from Nature Publishing Group.

but was visualized in lysosomes. The authors reasoned that endosomal retention of $\mathrm{CpG}-\mathrm{A}$ in $\mathrm{pDCs}$ provides a spatiotemporal regulation of TLR-9 signalling, necessary for IFN induction mediated by MyD88-IRF7. Indeed, artificially manipulating the transport of CpG-A in cDCs and macrophages, by complexing with cationic lipids, to resemble that of pDCs, resulted in the production of high levels of IFN- $a$ through the activation of the TLR-9-MyD88-IRF-7 pathway. Analogous manipulation of CpG-B - a ligand that does not normally activate this pathway in $\mathrm{pDCs}$ - had a similar effect.

Thus, pDCs seem to use a unique, although still enigmatic, mechanism to retain TLR-9-bound CPG-A in the endosomes to efficiently activate the MyD88-IRF-7 pathway and produce high levels of IFN. This spatiotemporal regulation might also explain how different types of unmethylated DNA activate distinct gene induction programmes by confining signalling pathways spatially. Collectively, the findings of Honda et al. have armed us with a better understanding of the interferon response, and will be critical to efficient therapeutic regulation of derailed immune responses.

MYRTO RAFTOPOULOU 